\title{
Sobrevida de pacientes con fibrosis pulmonar idiopática a la altura de Bogotá $(2640 \mathrm{~m})$
}

\author{
Survival in patients with idiopathie pulmonary \\ fibresis at the altitude of Bogetá $(2640 \mathrm{~m})$
}

\author{
Mauricio González-García, Julia Chamorro, Claudia Jaramillo, \\ Alejandro Casas, Darío Maldonado - Bogotá, D.C. (Colombia)
}

\section{Resumen}

Introducción: la sobrevida promedio de pacientes con fibrosis pulmonar idiopática (FPI) a nivel del mar es dedosatres años. Desconocemos su comportamiento en Bogotá, ciudad situada a gran altura (2.640 metros), donde hay mayor hipoxemia, factor asociado en la literatura con mal pronóstico. El objetivo del estudio es describir, en una cohorte de pacientes con FPI, la sobrevida y las características clínicas y funcionales en el momento del diagnóstico.

Material y métodos: pacientes con diagnóstico de FPI confirmado por biopsia o por criterios clínicos, radiológicos y funcionales. Se utilizó el análisis de sobrevida de Kaplan Meier y la prueba $\log$ rank.

Resultados: cuarenta pacientes, 50\% mujeres, con edad de $59.1 \pm 13$ añosy biopsia en $52.5 \%$. Al diagnóstico, $\mathrm{PaO}_{2}: 48.5 \pm 11.2 \mathrm{mmHg}, \mathrm{CVF}: 61.5 \pm 16.8 \%$, CPT: $66.1 \pm 11.7 \%, \mathrm{D}_{\mathrm{LCO}}: 39.5 \pm 12.4$ $\%$. La sobrevida desde el diagnóstico fue 42.0 meses (IC 95\% 25.3-58.7 meses) y desde el inicio de los síntomas 50.0 meses (IC 95\% 40.3-59.7 meses). No hubo diferencias en la sobrevida por sexo, historia de tabaquismo, forma de diagnóstico (biopsia o no biopsia), variables gasométricas y de función pulmonar. Hubo una menor sobrevida en mayores de 60 años (23.0 vs.72.0 meses, $\mathrm{p}=0.03)$.

Conclusiones: la sobrevida de pacientes con FPI en Bogotá con hipoxemia significativa al diagnóstico, fue similar a la descrita en estudios a nivel del mar. La edad mayor de 60 años se relacionó con mal pronóstico. (Acta Med Colomb 2014; 39: xx-xx).

Palabras clave: fibrosis pulmonar idiopática, sobrevida, altitud.

\section{Abstract}

Introduction: The survival rate of patients with idiopathic pulmonary fibrosis (IPF) at sea level is 2 to 3 years. There is no information at the altitude of Bogotá $(2.640 \mathrm{~m})$, where these patients have more severe hypoxemia, a factor assoeiated with worse prognosis. Our objective was to deseribe, in a cohort of patients with IPF living in Bogota, the elinieal and funetional eharacteristies at the diagnosisand its survival.

Methods: Patients with IPF eonfirmed by lung biopsy or by elinieal, tomographie, and funetionat eriteria. The Kaplan Meier plots and log rank test were used.

Results:Forty patients, $50 \%$ women, $59.1 \pm 13$ years old, and lung biopsy in $52.5 \%$. At the diagnosis: $\mathrm{PaO}_{z}: 48.5 \pm 11.2 \mathrm{mmHg}$, FVC: $61.5 \pm 16.8 \%$, TLC: $66.1 \pm 11.7 \%$, and $\mathrm{DL}_{\mathrm{GQ}}: 39.5 \pm 12.4 \%$. The survival time from the diagnosiswas 39.8 months (CI95\%, 29.3 to 50.3) and from the onset of symptoms was 51.7 months (CI95\%, 40.8 to 62.6). There weren't any differenees in survival aeeording to sex, smoking statts, diagnosis method (lung biopsy or not) or pulmonary funetion test. The survival in patients 60 years or older was lower than in those under $60(23.0 \mathrm{vs} .72 .0$ months, $\mathrm{p}=0.03)$.

Conelusion: The survival of patients with IPF living in Bogotá, a eity loeated at high altitude with severe hypoxemia at diagnosis, was similar to that deseribed at sea level. An age of 60 years or older was the only faetor related to a worse prognosis. (Acta Med Colomb 2014; 39: xx-xx).

Key words: idiopathie pulmonary fibrosis, survival, altitude
Dr. Mauricio González-García: Internista, Neumólogo, Epidemiólogo Clínico, Fundación Neumológica Colombiana; Dra. Julia Chamorro: Internista, Neumóloga, Fundación para el Cuidado del Corazón y del Pulmón; Dra. Claudia Jaramillo Villegas, MsC: Internista, Cardióloga, Magister en Epidemiología Clínica, Fundación Clínica Shaio; Dr. Alejandro Casas Herrera: Internista, Neumólogo, PhD, Fundación Neumológica Colombiana; Dr. Darío Maldonado Gómez: Internista, Neumólogo, Fundación Neumológica Colombiana. Bogotá D.C. (Colombia). Correspondencia. Dr. Mauricio González. Bogotá D.C. (Colombia).

E-mail: mgonzalez@neumologica.org Recibido: 19/XI/2012 Aceptado: 16/X/2013 


\section{Introducción}

La fibrosis pulmonar idiopática (FPI) es una enfermedad poco frecuente, cuya prevalencia varía, según diferentes estudios, entre 2 y 29 casos por 100.000 personas año en la población general (1). Su incidencia global se ha estimado en 4.6 por 100.000 personas año (2), 7.4 casos por 100.000 por año en mujeres y 10.7 en hombres (3). El diagnóstico se puede establecer por la presencia de criterios clínicos, tomográficos y funcionales más la exclusión de otras enfermedades o por biopsia pulmonar en algunos casos (1).

La FPI se caracteriza por un mal pronósticocon una mediana de sobrevida desde el diagnóstico entre 2 y 3 años en estudios realizados a nivel del mar $(1,4,5)$. Se han descrito biomarcadores, factores clínicos, tomográficos, patológicos y funcionales asociados con este mal pronóstico.Entre estos últimos, los principales son la capacidad vital, la capacidad pulmonar total, la difusión de monóxido de carbono y algunos parámetros de oxigenación como la saturación y la presión arterial de oxígeno en reposo o ejercicio $(1,5)$.

A la altura de Bogotá, una ciudad situada a gran altura (2 640 m), la disminución de la presión barométrica (560 $\mathrm{mmHg}$ ) condiciona una disminución de la presión inspirada, la presión alveolar y la presión arterial de oxígeno en sujetos normales (6-8) y en pacientes con enfermedades pulmonares crónicas. Debido a que la hipoxemia ha sido relacionada con pronóstico en la FPI, esta podría determinar cambios en la sobrevida de los pacientes. Como a esta altura no hay información sobre el pronóstico de la enfermedad, el objetivo del presente estudio fue describir, en una cohorte de pacientes con diagnóstico de FPI, la sobrevida y las características clínicas y funcionales al momento del diagnóstico.

\section{Material y métodos}

Estudio observacional de cohorte enpacientes mayores de 18 años con diagnóstico de primera vez (casos nuevos) de FPI. Se tomaron casos consecutivos atendidos en consulta externa y en hospitalización de la Fundación Neumológica Colombiana y la Fundación Cardioinfantil, institución de cuarto nivel desde 1999 hasta 2004. El diagnóstico de FPI se realizó de dos maneras según la guías internacionales vigentes al momento de iniciar el estudio: a) Pacientes con cuadro clínico, tomográfico y funcional compatible con FPI y biopsia pulmonar con hallazgos de neumonía intersticial usual y b) En ausencia de biopsia pulmonar, por la presencia de todos los criterios diagnósticos mayores y al menos tres de los cuatro criterios menores establecidos por la Sociedad Americana del Tórax (ATS) (4).

Criterios mayores. 1) Exclusión de otras causas de enfermedad pulmonar intersticial como toxicidad a medicamentos, exposición ambiental y enfermedades del tejido conectivo. 2) Estudios anormales de función pulmonar que incluyen evidencia de restricción (CVF reducida y frecuentemente aumento de la relación $\mathrm{VEF}_{1} / \mathrm{CVF}$ ) y/o deterioro del intercambio gaseoso (aumento de la $\mathrm{P}(\mathrm{A}-\mathrm{a}) \mathrm{O}_{2}$ en reposo o ejercicio o disminución de la $\mathrm{DL}_{\mathrm{CO}}$ ). 3) Anormalidades reticulares bibasales con mínimas opacidades en vidrio esmerilado en la tomografía computarizada de alta resolución. 4) Biopsia pulmonar transbronquial o lavado broncoalveolar (LBA) descartando diagnósticos alternativos.

Criterios menores. 1) Edad mayor de 50 años. 2) Comienzo insidioso de disnea con el ejercicio no explicada. 3) Duración de la enfermedad mayor de 3 meses. 4) Estertores inspiratorios bibasales (secos o tipo "velcro").

Se excluyeron pacientes que no cumplieron los criterios diagnóstico establecidos por la ATS (4) y sujetos con otras patologías pulmonares como asma, enfermedad pulmonar obstructiva crónica, secuelas de tuberculosis, cáncer. El estudio fue aprobado por el Comité de Ética de la institución.

\section{Evaluación clínicay funcional}

Se registraron datos de edad, sexo, talla, peso, índice de masa corporal, historia de tabaquismo (antecedente de consumo de al menos un paquete de cigarrillos/año), síntomas respiratorios con el tiempo de evolución y hallazgos al examen físico. Las pruebas de función pulmonar fueron realizadas en el Laboratorio de Fisiología de la Fundación Neumológica Colombiana: espirometría con broncodilatador, medición de la capacidad de difusión de monóxido de carbono $\left(\mathrm{D}_{\mathrm{L}, \mathrm{CO}}\right)$ por el método de respiración única y medición de volúmenes pulmonares por pletismografía enun V-MAX 229 - AUTOBOX (Sensormedics Inc, Yorba Linda, CA) según los criterios de la American Thoracic Society (ATS) y la European Respiratory Society (ERS) y se utilizaron las ecuaciones de referencia de Crapo (9-12). La muestra de gases arteriales se obtuvo por punción radial respirando aire ambiente (Fracción inspirada del 21\%).

\section{Análisis estadístico}

Las variables continuas se expresaron como promedios y desviación estándarsi tenían distribución normal o en caso contrario como mediana y rango intercuartílico (RIQ). Las variables cualitativas se expresaron como porcentajes. Para explorar diferencias entre los grupos con y sin biopsia pulmonar se utilizó la prueba $t$ de Student para las variables continuas y el $\chi^{2}$ para las variables cualitativas.

Se utilizó el método de Kaplan Meier para obtener gráficos y tiempo de sobrevida en la cohorte total de pacientes y estratificando por sexo, tabaquismo, grupo de edad, biopsia pulmonar y las variables de espirometría, volúmenes pulmonares y $\mathrm{D}_{\mathrm{L}, \mathrm{CO}}$. Para las pruebas de función pulmonar se utilizaron puntos de corte descritos en la literatura como factores pronósticosdel curso de la FPI $(1,13,14)$. El tiempo de sobrevida fue calculado desde el inicio de los síntomas y desde el diagnóstico hasta la muerte del paciente o fecha de censura. Los pacientes fueron censurados si estaban vivos en el último seguimiento. Se utilizó el log rank test para comparar las curvas de sobrevida entre los grupos. Un valor de $\mathrm{p}<0.05$ se consideró significativo. Se utilizó el paquete estadístico SPSS 15.0. 


\section{Resultados}

Se incluyeron 40 pacientes, 20 hombres y 20 mujeres con edad promedio de 59.2 \pm 13.4 años (29 a 85 años). La mediana de duración de síntomas antes del diagnóstico fue de 7.5 meses (RIQ 2 a 24 meses). De los 40 pacientes, 13 (32.5\%) tenían historia de tabaquismo. Los síntomas más frecuentes fueron disnea en 36 pacientes (90\%) y tos en 32 (80\%). En el examen físico todos los pacientes tenían estertores finos inspiratorios y 14 (35\%) tenían hipocratismo digital. Al momento del diagnóstico los pacientes tenían hipoxemia y desaturación en los gases arteriales, alteración restrictiva confirmada por volúmenes pulmonares y disminución de la difusión sin diferencias entre el grupo de sobrevivientes y el de no sobrevivientes (Tabla 1).

El diagnóstico fue establecido por biopsia pulmonar con un cuadro histológico de neumonía intersticial usual en 21 pacientes $(52.5 \%)$ y en los 19 restantes el diagnóstico se realizó por criterios clínicos, radiográficos y funcionales. No hubo diferencias entre estos grupos en la función pulmonar o los gases arteriales, pero los pacientes a quienes se les realizó biopsia pulmonar tenían una edad menor $(53.7 \pm 13.8$ vs. $65.2 \pm 10.1, \mathrm{p}<0.01)$ y una mediana demeses de síntomas menor (3.0 meses, RIQ 1.5 a 8.5 vs. 12.0 meses, RIQ 4 a $24 ; \mathrm{p}<0.01)$.En cuanto al tratamiento, 26 pacientes $(65 \%)$ recibieron esteroides sistémicos, 7 (17.5\%) ciclofosfamida, $7(17.5 \%)$ colchicina y $6(15 \%)$ azatioprina.
Se realizó un seguimiento promedio de 25.1 222.1 meses (mediana de20.5 meses, RIQ 4.0 a 24.0 meses) y hubo 21 muertes $(52.5 \%)$. La sobrevida promedio desde el inicio de los síntomas fue de 50.0 meses (IC 95\%, 40.3 - 59.7) y desde el diagnóstico de 42.0 meses (IC 95\%, 25.3-58.7) (Figura 1). No hubo diferencias en la sobrevida por sexo (hombres 44.0 meses, IC 95\% 11.8-76.2 Vs. mujeres 42.0 meses, IC 95\% 22.7-61.3; $\mathrm{p}=0.57$ ) (Figura 2), historia o no de tabaquismo (46.0 meses, IC 95\% 27.1-65.3 Vs. 36.0 meses, IC 95\% 23.9-48.6; $\mathrm{p}=0.38$ ) o entre los grupos con diagnóstico por biopsia o por criterios clínicos, radiográficos o funcionales (biopsia 44.0 meses, IC 95\% 19.4-68.6 Vs. no biopsia 30.0 meses, IC 95\% 18.8-41.2; p=0.88)(Figura 3). La sobrevida fue significativamente menor en los pacientes mayores de 60 años (23.0 meses, IC 95\% 8.0-38.0vs. 72.0 meses, IC $95 \%$ 26.8-117.2; $\mathrm{p}=0.03$ ) (Figura 4). No hubo diferencias en sobrevida al analizar las variables gasométricas y funcionales en el momento del diagnóstico.

\section{Discusión}

La sobrevida de pacientes adultos con fibrosis pulmonar idiopática (FPI) a la altura de Bogotá (2640 m), como hallazgo principal de este estudio,fue de 42.0 meses desde el momento del diagnóstico, similar a lo publicadoen estudios a nivel del mar. La edad mayor de 60 años se asoció significativamente apeor pronósticode la enfermedad. No

Tabla 1. Características clínicas y pruebas de función pulmonar.

\begin{tabular}{|c|c|c|c|c|}
\hline & Grupo total $(N=40)$ & Sobrevivientes $(\mathrm{N}=19)$ & No sobrevivientes $(\mathrm{N}=21)$ & $\mathbf{p}$ \\
\hline Edad. años & $59.2 \pm 13.4$ & $56.3 \pm 11.9$ & $61.8 \pm 14.3$ & 0.20 \\
\hline IMC. $\mathrm{Kg} / \mathrm{m}^{2}$ & $26.3 \pm 3.1$ & $26.3 \pm 2.8$ & $26.3 \pm 3.5$ & 0.97 \\
\hline $\mathrm{Hb} . \mathrm{gr} / \mathrm{dL}$ & $17.9 \pm 9.8$ & $18.8 \pm 12.3$ & $17.3 \pm 7.6$ & 0.67 \\
\hline $\mathrm{pH}$ & $7.41 \pm 0.05$ & $7.41 \pm 0.03$ & $7.41 \pm 0.06$ & 0.85 \\
\hline $\mathrm{PaCO}_{2} \cdot \mathrm{mmHg}$ & $31.3 \pm 4.8$ & $31.0 \pm 5.8$ & $31.6 \pm 3.9$ & 0.72 \\
\hline $\mathrm{PaO}_{2} \cdot \mathrm{mmHg}$ & $48.5 \pm 11.2$ & $48.3 \pm 11.9$ & $48.7 \pm 10.8$ & 0.92 \\
\hline $\mathrm{HCO}_{3} \cdot \mathrm{mEq} / \mathrm{L}$ & $20.1 \pm 3.9$ & $19.8 \pm 3.8$ & $20.4 \pm 4.0$ & 0.71 \\
\hline $\mathrm{SaO}_{2} \cdot \%$ & $82.5 \pm 12.6$ & $81.2 \pm 15.2$ & $83.9 \pm 9.6$ & 0.57 \\
\hline $\mathrm{P}(\mathrm{A}-\mathrm{a}) \mathrm{O}_{2} \cdot \mathrm{mmHg}$ & $20.0 \pm 12.5$ & $20.0 \pm 14.6$ & $19.9 \pm 10.8$ & 0.98 \\
\hline CVF. \% & $61.5 \pm 16.8$ & $62.5 \pm 16.9$ & $60.4 \pm 17.1$ & 0.72 \\
\hline $\mathrm{VEF}_{1} \cdot \%$ & $66.5 \pm 18.1$ & $65.9 \pm 16.8$ & $67.1 \pm 19.8$ & 0.85 \\
\hline $\mathrm{VEF}_{1} / \mathrm{CVF}$ & $87.2 \pm 7.1$ & $86.4 \pm 6.7$ & $88.1 \pm 7.5$ & 0.48 \\
\hline СРТ. \% & $66.2 \pm 11.7$ & $68.2 \pm 9.9$ & $64.8 \pm 12.9$ & 0.47 \\
\hline VR. \% & $79.6 \pm 29.6$ & $83.6 \pm 16.3$ & $76.9 \pm 36.3$ & 0.52 \\
\hline VR/CPT & $42.1 \pm 13.6$ & $44.1 \pm 15.7$ & $40.7 \pm 12.3$ & 0.54 \\
\hline CRF. \% & $75.3 \pm 18.5$ & $74.6 \pm 10.5$ & $75.8 \pm 22.8$ & 0.85 \\
\hline VA. $\%$ & $58.4 \pm 13.1$ & $58.9 \pm 12.5$ & $57.9 \pm 13.9$ & 0.83 \\
\hline DLCO. $\%$ & $39.5 \pm 12.4$ & $36.9 \pm 13.4$ & $41.9 \pm 11.4$ & 0.28 \\
\hline DLCO/VA. $\%$ & $66.8 \pm 18.6$ & $60.7 \pm 18.5$ & $72.1 \pm 17.5$ & 0.08 \\
\hline
\end{tabular}




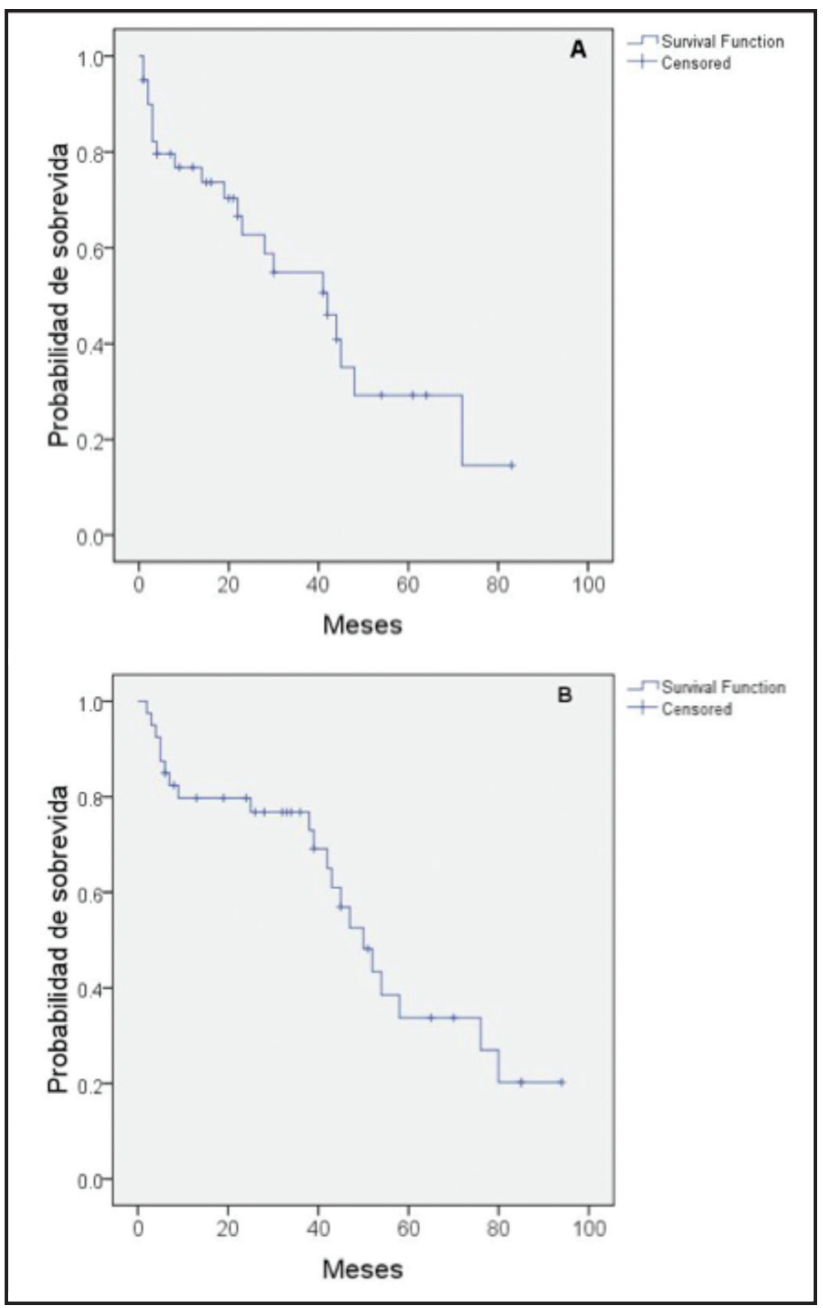

Figura 1. Curvas de Kaplan-Meier de sobrevida en meses. A: desde el diagnóstico (42 meses; IC 95\%, 25.3 - 58.7.3). B: desde el inicio de los síntomas (50.0 meses; IC 95\%, 40.3-59.7).

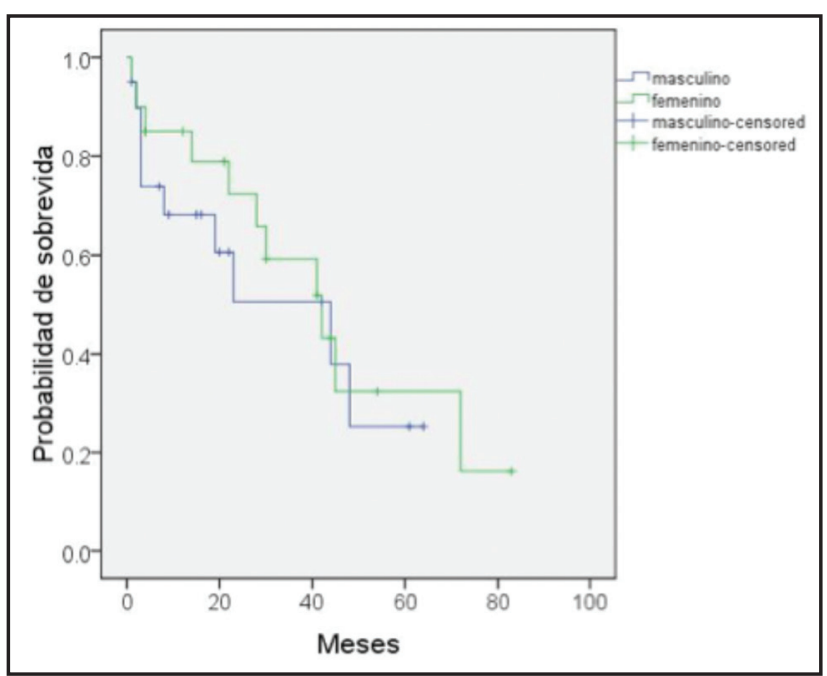

Figura 3. Curva de Kaplan-Meier según método diagnóstico. No hubo diferencias significativas entre la mediana de sobrevida entre los pacientes diagnóstico sin biopsia (30.0 meses; IC 95\%,18.8-41.2) Vs. los pacientes con biopsia pulmonar (44.0 meses; IC 95\%,19.4-68.6) $P=0.88$

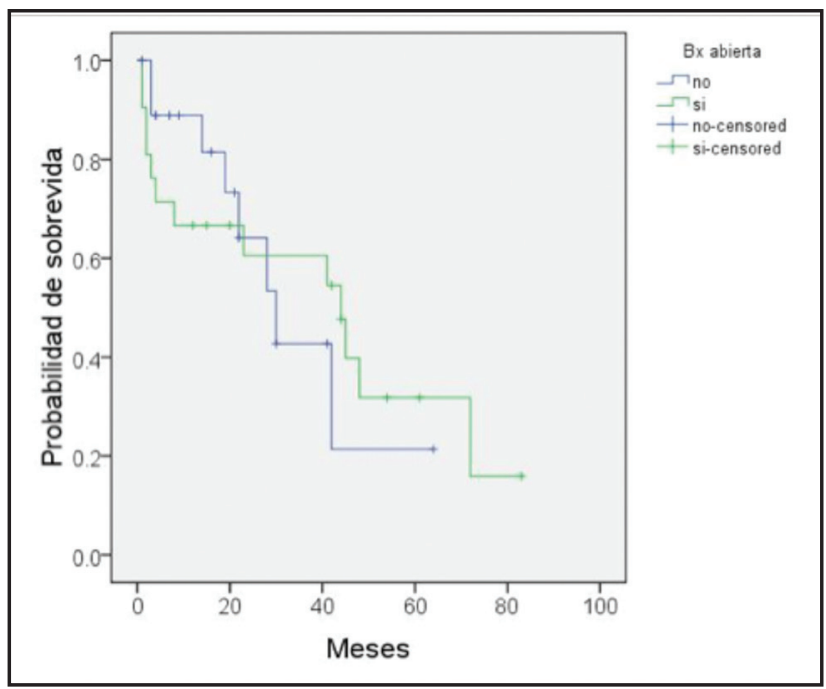

Figura 2. Curvas de Kaplan-Meier según sexo. No hubo diferencias significativas entre la mediana de sobrevida entre hombres (44.0 meses; IC 95\%,11.8 - 76.2) vs. mujeres (42.0 meses; IC 95\%, $22.7-62.3), p=0.57$.

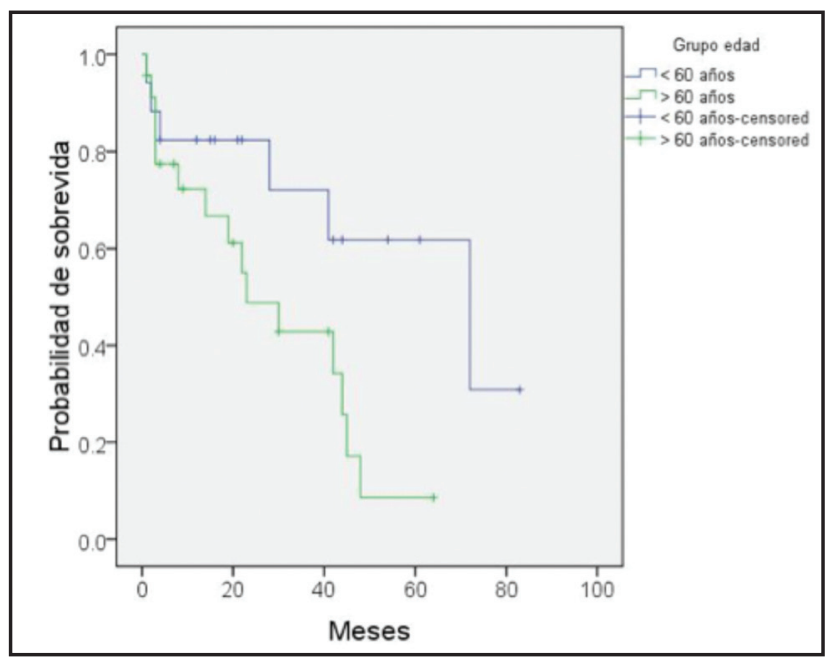

Figura 4. Curva de Kaplan-Meier según grupos de edad. La mediana de sobrevida fue significativamente mayor en los pacientes menores de 60 años (72.0 meses, IC 95\% 26.8 117.2) Vs los mayores de 60 años (23.0 meses, IC 95\% 8.0 - 38.0 vs), $p=0.03$.

hubo ninguna relación entre la sobrevida con las variables funcionales en reposo, el sexo o la historia de tabaquismo.

La FPI se caracteriza por su mal pronóstico con una mediana de sobrevida luego del diagnóstico entre dos y tres años en estudios realizados a nivel del mar (15-19), similar a los hallazgos del presente estudio. La sobrevida de51.7 meses desde el comienzo de los síntomas encontrada en nuestro estudio es mayora lo descrito previamente. La mediana de inicio de síntomas antes del diagnóstico reportada en la literatura es de uno a dos años $(15,17,19,20)$, significativamente mayor a la encontrada en estos pacientes de 7.5 meses. Esto sugiere que los pacientes de la cohorte de este estudio consultaron más tempranamente, lo cual podría explicarse poruna mayor percepción de síntomas en las etapas iniciales de la enfermedad. 
En nuestro estudio, en concordancia con la literatura, la edad mayor a 60 años al momento del diagnostico se asociócon mal pronóstico.La FPI es una enfermedad predominantemente de población mayor, con una mediana de edad al momento del diagnóstico de 66 años (4). Actualmente se considera que la edad avanzada es un factor de mal pronóstico $(13,21)$. No encontramos diferencias en la sobrevida entre hombres y mujeres, hallazgo similar alodescrito enotros estudios $(13,21)$. Aunque los datos en la literatura no son consistentes, recientemente se ha descrito un mejor pronóstico en mujeres con PFI al ajustar por edad, tabaquismo, variables funcionales en reposo y capacidad de ejercicio medida en la caminata de seis minutos (22). En esta cohorte no encontramos diferencias de sobrevida según la historia de tabaquismo, lo cual se había sugerido inicialmentecomo un factor de buen pronóstico de la FPI (21), pero estudios recientes descartan esta asociación después de ajustar el análisis por variables de severidad de la enfermedad (23).

Aunque no tuvimos confirmación histológica en todos los pacientes, se acepta que luego de descartar causas conocidas de neumonía intersticial usual, se puede establecer el diagnóstico de FPI con criterios característicos clínicos, tomográficos y funcionales (1). Adicionalmente al comparar los casos con y sin biopsia pulmonar, las únicas diferencias entre los grupos fueron la menor edad y menor tiempo de inicio de los síntomas en los pacientes con biopsia, sin diferencias en síntomas, hallazgos clínicos, funcionales o en la sobrevida, lo que sustentael diagnóstico de FPI realizado sin biopsia. El hallazgo de menor edad del grupo de pacientes con biopsia es similar a lo descrito en el estudio de Hubbard (24) en el cual solamente el $12.7 \%$ de los pacientes tuvieron confirmación de la FPI por biopsia abierta. En otra cohorte de 115 paciente con FPI, solamente se realizó biopsia pulmonar en $44(38 \%)$ sin encontrar diferencias en sobrevida entre los grupos con y sin biopsia (14), similar a nuestros resultados.

A diferencia de varias publicaciones previas no encontramos relación entre la sobrevida y los resultados de los gases arteriales y las pruebas de función pulmonar al momento del diagnóstico. Como era de esperarse, en este estudio realizado en Bogotá, una ciudad situada a gran altura (2640 m), encontramos niveles significativos de hipoxemia, pero no pudimos confirmar que esto afectara la sobrevida de los pacientes como se ha sugerido en estudios a nivel del mar (25). El no encontrar relación entre el grado de hipoxemia y la sobrevida sugiere la presencia de mecanismos de adaptación a la altitud, pero se requieren estudios adicionales con un mayor número de pacientespara confirmar esta teoría. Dentro de las variables funcionales, la que más se ha asociado al pronóstico ha sido la DLCO, sugiriéndose, en la mayoría de los estudios, que un valor inferior a un punto de corte de $40 \%$ del valor predicho se relaciona con peor pronóstico $(20,26$, 27). Otras factores que se han relacionado con mortalidad en la FPI son algunas variables en ejercicio como el consumo de oxígeno ola distancia caminada y los cambios en saturación en la caminata de seis minutos (28-31), pero la tendencia actual es utilizar no solamente las variables funcionales al momento del diagnóstico, sino las variaciones en el tiempo y los puntajes compuestos incluyendo variables histológicas y tomográficas $(20,21,30,32-36)$.

Destacamos del presente estudio que es la primera descripción sobre la sobrevida de la FPI en una ciudad a gran altura,con seguimiento de un número significativo de pacientes de una enfermedad poco frecuente, lo que aporta al conocimiento del comportamiento de esta enfermedadala altura de Bogotá o alturas similares.Una limitación del estudio es el bajo número de pacientes incluidos, que aunque permite describir en forma adecuada la sobrevida, no permite concluir sobre los factores asociados a mortalidad de esta enfermedad. Otra limitación es el no contar con información sobre variables en ejercicio y el cambio de la función pulmonar en el tiempo. Se requieren otros estudios con un número mayor de pacientes que permitan un análisis multivariado de factores pronósticos, incluyendo las variables funcionales mencionadas y hallazgos en la tomografía o en la histología.

En conclusión, los resultados de este trabajo muestran que la sobrevida de pacientes con fibrosis pulmonar idiopática en Bogotá (2640 m) es similar a la publicada en estudios realizados a nivel del mar. La variable que se relacionó con peor pronóstico fue la edad al momento del diagnóstico mayor de 60 años. Se deben realizar otros estudios con un mayor número de pacientes para evaluar los factores pronósticos de esta enfermedad a la altura de Bogotá.

\section{Financiación}

Esta investigación se realizó con recursos de la Fundación Neumológica Colombiana y no se recibió aportes de otra entidad.

\section{Conflictos de interes}

Los autores no informan conflictos de interés con el presente trabajo.

\section{Referencias}

1. Raghu G, Collard HR, Egan JJ, Martinez FJ, Behr J, Brown KK et al. An official ATS/ERS/JRS/ALAT statement: idiopathic pulmonary fibrosis: evidencebased guidelines for diagnosis and management. Am J Respir Crit Care Med 2011; 183: $788-824$

2. Gribbin J, Hubbard RB, Le Jeune I, Smith CJP, West J, Tata LJ. Incidence and mortality of idiopathic pulmonary fibrosis and sarcoidosis in the UK. Thorax 2006; 61: 980-5.

3. Coultas DB, Zumwalt RE, Black WC, Sobonya RE. The epidemiology of interstitial lung diseases. Am J Respir Crit Care Med 1994; 150: 967-72.

4. American Thoracic Society. Idiopathic pulmonary fibrosis: diagnosis and treatment. International consensus statement. American Thoracic Society (ATS), and the European Respiratory Society (ERS). Am J Respir Crit Care Med 2000; 161: 646-64.

5. Ley B, Collard HR, King TE. Clinical Course and Prediction of Survival in Idiopathic Pulmonary Fibrosis. Am J Respir Crit Care Med 2011; 183: 431-40.

6. Acevedo L, Solarte I. Gasimetría arterial en adultos sanos a nivel de Bogotá Acta Med Colomb 1984; 9: 7-14.

7. Restrepo J, Reyes P, Vásquez P. Gasimetria arterial y alveolar en adultos sanos a nivel de Bogotá. Acta Med Colomb 1982; 7: 461-6.

8. Roa J, de Arroyo S, Salcedo MC, Maldonado D. Gasimetría arterial a diferentes edades en Bogotá. Acta Med Colomb 1988; 13S: 415A(C217).

9. Crapo RO, Morris AH, Gardner RM. Reference spirometric values using techniques and equipment that meet ATS recommendations. Am Rev Respir Dis 1981; 123: 659-64.

10. American Thoracic Society. Lung function testing: selection of reference values and interpretative strategies. Am Rev Respir Dis 1991; 144: 1202-18. 
11. American Thoracic Society. Standardization of Spirometry, 1994 Update. Am J Respir Crit Care Med 1995; 152: 1107-36.

12. American Thoracic Society. Single-breath carbon monoxide diffusing capacity (transfer factor). Recommendations for a standard technique-1995 update. Am J Respir Crit Care Med 1995; 152: 2185-98.

13. Erbes R, Schaberg T, Loddenkemper R. Lung function tests in patients with idiopathic pulmonary fibrosis. Are they helpful for predicting outcome? Chest 1997; 111: 51-7.

14. Mogulkoc N, Brutsche M, Bishop P, Greaves S, Horrocks A, Egan J. Pulmonary Function in Idiopathic Pulmonary Fibrosis and Referral for Lung Transplantation. Am J Respir Crit Care Med 2001; 164: 103-8.

15. King T, Schwarz M, Brown K, Tooze J, Colby T, Waldron J et al. Idiopathic Pulmonary Fibrosis: Relationship between Histopathologic Features and Mortality. Am J Respir Crit Care Med 2001; 164: 1025-32.

16. Rudd RM, Prescott RJ, Chalmers JC, Johnston IDA. British Thoracic Society Study on cryptogenic fibrosing alveolitis: response to treatment and survival. Thorax 2007; 62: 62-6.

17. Nicholson A, Colby T, Dubois R, Hansell D, Wells A. The Prognostic Significance of the Histologic Pattern of Interstitial Pneumonia in Patients Presenting with the Clinical Entity of Cryptogenic Fibrosing Alveolitis. Am J Respir Crit Care Med 2000; 162: 2213-7.

18. Flaherty KR, Toews GB, Travis WD, Colby TV, Kazerooni EA, Gross BH et al. Clinical significance of histological classification of idiopathic interstitial pneumonia. Eur Respir J 2002; 19: 275-83.

19. Bjoraker J, Ryu J, Edwin M, Myers J, Tazelaar H, Schroeder D et al. Prognostic Significance of Histopathologic Subsets in Idiopathic Pulmonary Fibrosis. Am J Respir Crit Care Med 1998; 157: 199-203.

20. Collard HR, King TE, Bartelson BB, Vourlekis JS, Schwarz MI, Brown KK. Changes in Clinical and Physiologic Variables Predict Survival in Idiopathic Pulmonary Fibrosis. Am J Respir Crit Care Med 2003; 168: 538-42.

21. King T, Tooze J, Schwarz M, Brown K, Cherniak R. Predicting Survival in Idiopathic Pulmonary Fibrosis: Scoring System and Survival Model. Am J Respir Crit Care Med 2001; 164: 1171-81.

22. Han MK, Murray S, Fell CD, Flaherty KR, Toews GB, Myers J et al. Sex differences in physiological progression of idiopathic pulmonary fibrosis. Eur Respir J 2008; 31: 1183-8.

23. Antoniou KM, Hansell DM, Rubens MB, Marten K, Desai SR, Siafakas NM et al. Idiopathic Pulmonary Fibrosis: Outcome in Relation to Smoking Status. Am J Respir Crit Care Med 2008; 177: 190-4.

24. Hubbard R, Johnston I, Britton J. Survival in patients with cryptogenic fibrosing alveolitis: a population-based cohort study. Chest 1998; 113: 396-400.

25. Timmer S.J, Karamzadeh AM, Yung GL, Kriett J, Jamieson SW, Smith CM. Predicting survival of lung transplantation candidates with idiopathic interstitial pneumonia: does $\mathrm{PaO}_{2}$ predict survival? Chest 2002; 122: 779-84.

26. Egan JJ, Martinez FJ, Wells AU, Williams T. Lung function estimates in idiopathic pulmonary fibrosis: the potential for a simple classification. Thorax 2005 60: $270-3$

27. Hamada K, Nagai S, Tanaka S, Handa T, Shigematsu M, Nagao T et al. Significance of Pulmonary Arterial Pressure and Diffusion Capacity of the Lung as Prognosticator in Patients With Idiopathic Pulmonary Fibrosis. Chest 2007; 131: $650-6$

28. Hallstrand TS, Boitano LJ, Johnson WC, Spada CA, Hayes JG, Raghu G. The timed walk test as a measure of severity and survival in idiopathic pulmonary fibrosis. Eur Respir J 2005; 25: 96-103.

29. Lederer DJ, Arcasoy SM, Wilt JS, D’Ovidio F, Sonett JR, Kawut SM. SixMinute-Walk Distance Predicts Waiting List Survival in Idiopathic Pulmonary Fibrosis. Am J Respir Crit Care Med 2006; 174: 659-64.

30. Flaherty KR, Andrei AC, Murray S, Fraley C, Colby TV, Travis WD et al. Idiopathic Pulmonary Fibrosis: prognostic value of changes in physiology and six-minute-walk test. Am J Respir Crit Care Med 2006; 174: 803-9.

31. Lama VN, Flaherty KR, Toews GB, Colby TV, Travis WD, Long Q et al. Prognostic Value of Desaturation during a 6-Minute Walk Test in Idiopathic Interstitial Pneumonia. Am J Respir Crit Care Med 2003; 168: 1084-90.

32. Hanson D, Winterbauer RH, Kirtland SH, Wu R. Changes in pulmonary function test results after 1 year of therapy as predictors of survival in patients with idiopathic pulmonary fibrosis. Chest 1995; 108: 305-10.

33. Jegal Y, Kim DS, Shim TS, Lim CM, Do Lee S, Koh Y et al. Physiology Is a Stronger Predictor of Survival than Pathology in Fibrotic Interstitial Pneumonia. Am J Respir Crit Care Med 2005; 171: 639-44.

34. Latsi PI, du Bois RM, Nicholson AG, Colby TV, Bisirtzoglou D, Nikolakopoulou A et al. Fibrotic Idiopathic Interstitial Pneumonia: The Prognostic Value of Longitudinal Functional Trends. AmJ Respir Crit Care Med 2003; 168: 531-7.

35. Wells AU, Desai SR, Rubens MB, Goh NSL, Cramer D, Nicholson AG et al. Idiopathic Pulmonary Fibrosis: A Composite Physiologic Index Derived from Disease Extent Observed by Computed Tomography. Am J Respir Crit Care Med 2003; 167: 962-9.

36. Flaherty KR, Mumford JA, Murray S, Kazerooni EA, Gross BH, Colby TV et al. Prognostic Implications of Physiologic and Radiographic Changes in Idiopathic Interstitial Pneumonia. AmJ Respir Crit Care Med 2003; 168: 543-8. 